\title{
Publikationsbias durch Datenduplikation und Datenselektion
}

P. Kleist

Ein Problem der evidenzbasierten Medizin besteht im sogenannten Publikationsbias. Während das Nichtpublizieren von Studienergebnissen in jüngster Vergangenheit ausreichend thematisiert wurde, finden andere Aspekte des vielschichtigen Publikationsbias nicht ausreichend Beachtung. Zwei davon werden in diesem Beitrag genauer betrachtet: das Duplizieren sowie die selektive Darstellung von Studiendaten in Publikationen. Deren Einfluss auf die Evidenzlage zu einer medizinischen Fragestellung, insbesondere im Hinblick auf Nutzen/Risiken- und Kosten/Nutzen-Analysen einer therapeutischen Intervention, darf nicht unterschätzt werden. Wirksame Massnahmen gegen diese Formen des Publikationsbias könnten sein: 1. eine verstärkte Selbstregulierung sowohl der Ärzteschaft als auch der pharmazeutischen Industrie; 2. die simultane Einreichung von Publikationsmanuskripten und der zugrundeliegenden Studienprotokolle; 3. die Einrichtung eines öffentlich zugänglichen und zentralen Studienregisters, durch das Studien obligat zu erfassen sind.

Korrespondenz:

Dr. med. Peter Kleist

Novartis Pharma Schweiz AG

Monbijoustrasse 118

CH-3001 Bern

E-Mail:

peter.kleist@pharma.novartis.com
Ein wesentlicher Anteil klinischer Studien wird nie publiziert. Die Ärzteschaft ist sich dessen heute allgemein bewusst - nicht zuletzt durch halt in den Medien geschenkt wurde. Auch die Tatsache, dass Studien mit nicht signifikantem oder bezüglich der Fragestellung negativem Ergebnis - wenn überhaupt - mit erheblicher Verzögerung veröffentlicht werden [1, 2], kann inzwischen als bekannt vorausgesetzt werden. Die Ursachen sind vielfältig: Das Publikationsinteresse mancher Wissenschaftler erlahmt [3], die pharmazeutische Industrie ist nicht immer bereit, ungünstige Daten für ihre Produkte einer breiten Leserschaft zugänglich zu machen [4] und das Interesse führender biomedizinischer Zeitschriften, Studien mit nicht signifikantem Ausgang zu publizieren, ist immer noch eingeschränkt [5]. Die daraus resultierende Überrepräsentation «positiver» Studien kann die tatsächliche Datenlage und Evidenzlage zu einer medizinischen Fragestellung verzerren.

Diesem sogenannten Publikationsbias liegt somit ein Verhalten zugrunde, das als wissenschaftliches Fehlverhalten eingestuft wird [6]. Tonks bringt es in einem Editorial des British Medical Journals auf den Punkt: «These biases are not just inconvenient, they cost lives» [7]. Verschiedene Richtlinien oder Kodizes haben daraufhin in jüngster Zeit dieses Thema aufgegriffen, um individuelles Fehlverhalten der unterschiedlichen, am Publikationsprozess beteiligten Pardie hohe Aufmerksamkeit, die diesem Sachver- teien im Sinne einer ethisch-motivierten Selbstregulierung abzustellen. Artikel 27 der revidierten Fassung der Deklaration von Helsinki hat die Publikation positiver wie negativer Studienergebnisse bereits vor vier Jahren zum ethischen Grundprinzip erklärt [8]. Die 2002 durch die Schweizerische Akademie der Medizinischen Wissenschaften (SAMW) herausgegebenen und an die Ärzteschaft adressierten Empfehlungen zur Zusammenarbeit Ärzteschaft-Industrie sprechen von der «Pflicht, Versuchsergebnisse zu veröffentlichen» [9]. Der zu Beginn 2004 in Kraft gesetzte Verhaltenskodex der pharmazeutischen Industrie in der Schweiz [10] konstatiert das grundsätzliche Publikationsrecht der Studienärzte unabhängig von den Interessen des Sponsors, d. h. unabhängig vom Ausgang einer Studie. Und die Richtlinien für eine gute Publikationspraxis [11], herausgegeben vom «Committee on Publication Ethics» mit Vertretern führender britischer biomedizinischer Zeitschriften, fordern die Publikation auch von Studien mit negativem Ausgang (insofern sie die anerkannten Kriterien wissenschaftlicher Qualität erfüllen).

Der Publikationsbias beschränkt sich jedoch nicht auf die Nichtveröffentlichung von Studienergebnissen, sondern beinhaltet weitere Aspekte, denen in der allgemeinen Diskussion über das Thema vielleicht zu wenig Beachtung geschenkt und die von den bestehenden ethischen Kodizes der Ärzte und der Industrie bisher nicht explizit aufgegriffen wurden. Dennoch können sie ebenso die Evidenzlage zu einer medizinischen Fragestellung potentiell verzerren. Auf zwei dieser Aspekte soll im folgenden aufmerksam gemacht werden: Die Duplizierung von Daten durch multiple oder redundante Publikationen und die selektive, d.h. unvollständige oder in bezug auf Fragestellungen und Endpunkte im Studienprotokoll inkonsistente Darstellung von Studienergebnissen.

\section{Datenduplikation}

In einer Veröffentlichung im British Medical Journal [12] verglich eine schwedische Forschergruppe 42 placebokontrollierte klinische Studien 
zu fünf verschiedenen selektiven Serotonin-Wiederaufnahmehemmern, die der schwedischen Arzneimittelbehörde im Rahmen von Zulassungsanträgen vorgelegt wurden, mit den im Laufe der Jahre veröffentlichten Daten. Auffallend war generell die Mehrfachverwertung von Daten durch Doppelpublikationen und die mehrfache Einbeziehung von Daten in gepoolte Auswertungen. Für ein Arzneimittel erschienen drei Originalpublikationen (mit positivem Studienausgang) jeweils zweimal. Gepoolte Analysen zu diesem Arzneimittel griffen überwiegend auf die positiven Studien zurück und schlossen die duplizierten Daten mit ein, ohne dass entsprechende Querverweise gemacht wurden. Dies ist nur ein Beispiel aus der jüngsten Vergangenheit, aber es finden sich viele in der Literatur. Im Jahr 1996 wurden von einer kanadischen Gruppe 20 publizierte randomisierte doppelblinde Studien zu einem Antipsychotikum identifiziert, denen jedoch lediglich zwei grössere und sieben kleinere Studien zugrunde lagen [13]. Eine solche Zerlegung der gleichen Studie in einzelne Datenpakete wurde bereits 1986 von Huth als «Salami Science» beschrieben [14]. Ein anderes Beispiel ist die parallele Einreichung des nahezu gleichen Manuskriptes sowohl beim Journal of the American College of Cardiology als auch beim American Heart Journal, welches anschliessend von beiden Zeitschriften in Unkenntnis des Duplikates abgedruckt wurde [15, 16]. Die einzigen inhaltlichen Unterschiede bestanden in der Anzahl der Patienten in der Kontrollgruppe und der Anzahl der Tabellen. Eine weitere Studie zu einem nichtsteroidalen Entzündungshemmer erschien mit einem Abstand von fünf Jahren sogar zweimal in demselben Journal $[17,18]$.

Wie häufig kommt die Datenduplikation durch multiple oder redundante Publikationen tatsächlich vor? Das Committee on Publication Ethics (COPE) in Grossbritannien erhält pro Jahr etwa 200 Anzeigen zu Publikationsverstössen unterschiedlicher Art [19]. Von den durch COPE zwischen Juli 2001 und September 2003 neu bearbeiteten 67 Fällen wissenschaftlichen Fehlverhaltens beziehen sich allein 12 Fälle auf Datenduplizierung [20]. Die Dunkelziffer scheint jedoch deutlich höher zu sein: Schätzungen zufolge betreffen redundante Darstellungen jeden fünften bis sechsten Artikel in medizinischen Fachzeitschriften [21, 22].

Was sind die Gründe für ein solches Verhalten? Es ist die Maximierung des Publikationsauswurfs von ein und derselben Datenbank zur Förderung der Karriere, zur Erzielung weiterer finanzieller Förderung und zur einseitigen
Promotion eines Arzneimittels [23]. Dies verwundert insofern nicht, als Berufungskommissionen an medizinischen Fakultäten die Kandidaten immer noch häufig an der Anzahl ihrer Publikationen und der Summe ihrer Impact-Faktoren beurteilen [24, 25]. Der Impact Factor, im Grunde nur eine Grösse zur Ermittlung der Qualität eines Journals, wird oftmals herangezogen, um die Forschungsleistung von Arbeitsgruppen, Instituten und Kliniken, mitunter sogar Fakultäten und Universitäten vergleichend zu messen. Daher lautet die Devise noch immer: «Publish or perish». Auf der Suche nach brauchbaren Kriterien sowohl für Berufungsverfahren als auch für eine gerechte Verteilung limitierter Forschungsmittel ist der Impact Factor heute jedoch zunehmend umstritten [25-27]. Und da für den Erfolg eines Arzneimittels im kompetitiven Pharmamarkt der sogenannte «Share of Noise» eine wichtige Grösse ist, decken sich die Interessen von Wissenschaftlern und von pharmazeutischen Unternehmen, wie die Beispiele in diesem Abschnitt zeigen.

Die Identifikation von redundanten Daten in der Literatur fällt in der Regel schwer, weil sich Datenduplikate oftmals hinter unterschiedlichen Autoren, unterschiedlichen Patientenselektionskriterien oder unterschiedlichen Patientenzahlen verbergen. Der Leser erhält unter Umständen ein falsches Bild von der tatsächlichen Wirksamkeit oder Sicherheit einer therapeutischen Intervention, vor allem, wenn redundante Information in Metaanalysen einfliesst. In einer Fallstudie wurden 84 Studien zu einem 5HT3Rezeptorantagonist analysiert, die zwischen 1991 und 1996 publiziert wurden [28]. Alle Studien untersuchten die Wirksamkeit der Substanz bezüglich des Auftretens postoperativen Erbrechens; insgesamt wurden nahezu 12000 Patienten mit dem 5HT3-Rezeptorantagonisten behandelt. Es stellte sich heraus, dass 14 dieser Studien bzw. die Daten von mehr als 3300 behandelten Patienten Duplikate darstellten. Die Metaanalyse unter ausschliesslicher Betrachtung nicht duplizierter Originalstudien ergab eine Number needed to treat (NNT) von 9,5; bei einer Metaanalyse unter zusätzlicher Berücksichtigung der Duplikate verbesserte sich die NNT auf 4,9. Dies ist eine Fallstudie, die in vollem Bewusstsein mit duplizierten Daten umgeht. Autoren von Metaanalysen stossen jedoch wiederholt auf Schwierigkeiten, redundante Daten $\mathrm{zu}$ identifizieren und duplizierte Information kann tatsächlich in publizierte Metaanalysen einfliessen, ohne dass sich die Autoren dieses Umstandes bewusst sind $[29,30]$. 
Die mehrmalige Veröffentlichung von Daten ist nur dann legitim, wenn sie an eine andere Leserschaft gerichtet ist, z. B. durch Übersetzung des Manuskripts in eine andere Sprache für Ärzte in einem anderen Land - immer vorausgesetzt, dass die Zustimmung beider Zeitschriften vorliegt und damit Copyrights gewahrt werden, die Zweitpublikation als solche deklariert und eine klare Bezugnahme auf die Originalpublikation gegeben ist [11, 31].

\section{Datenselektion}

In einer Veröffentlichung von Chan und Mitarbeitern im Journal of the American Medical Association wurden 122 Publikationen randomisierter Studien analysiert, deren zugrundeliegenden Studienprotokolle 1994 und 1995 von zwei dänischen Ethikkommissionen begutachtet wurden [32]. Die Zielsetzung bestand darin, erstmals systematisch die Vollständigkeit und Konsistenz der in den Publikationen erwähnten Studienendpunkte - im Abgleich mit den Protokollen - zu beurteilen. Es zeigte sich, dass im Durchschnitt 50\% der Wirksamkeits- und 65\% der Sicherheitsvariablen in den Publikationen unvollständig wiedergegeben wurden. Statistisch signifikante Ergebnisse wurden mit einer deutlich höheren Rate vollständig berichtet als nicht signifikante. $62 \%$ der untersuchten Studien wiesen darüber hinaus mindestens einen primären Endpunkt auf, der entweder neu eingeführt, verändert oder weggelassen wurde. Publikationen sind also häufig nicht nur unvollständig, sondern weisen oftmals auch Inkonsistenzen zu den Studienprotokollen auf. Primäre Endpunkte mutierten häufig zu sekundären Endpunkten oder umgekehrt, und mitunter wurden sogar Daten als primäres Studienergebnis präsentiert, deren Analyse nicht einmal im Protokoll vorgesehen war [32].

Auch in der bereits erwähnten schwedischen Arbeit über Serotonin-Wiederaufnahmehemmer [12] wurden die Publikationen mit den ursprünglichen Studienprotokollen verglichen. Da sich der Vergleich nicht nur auf die Studienendpunkte beschränkte, fielen zusätzliche Aspekte einer selektiven Datendarstellung auf: Mitunter wurden die Daten entweder nur als Intentionto-treat- oder Per-Protocol-Analyse dargestellt, je nach dem, welcher statistische Ansatz vorteilhafter für eine Intervention war. Patienten, die aus einer Studie ausschieden oder schwer auswertbar waren, wurden in der Publikation häufig nicht erwähnt. Und um ein Beispiel für einen konkreten Fall zu geben: Im Jahre 2000 erschien eine
Publikation zu 6-Monats-Follow-up-Daten von zwei Studien mit einem COX-2-Inhibitor [33], die in der Darstellung der Ergebnisse nicht nur erheblich vom Studienprotokoll abwich (bezüglich der Behandlungsdauer und der analysierten Endpunkte) [34], sondern darüber hinaus zum Zeitpunkt der Einreichung des Manuskriptes bereits bekannte und bezüglich des Arzneimittelrisikos relevante Informationen unerwähnt liess $[35,36]$. Die in der Publikation vorgenommene Nutzen/Risiko-Bewertung war im Grunde unbrauchbar, weil sie auf selektiven Teildaten beruhte, die weder der gesamten Datenlage noch den Vorgaben für die statistische Auswertung entsprachen.

Man kann davon ausgehen, dass der «Schönung» von Studienergebnissen durch Datenselektion die gleichen Beweggründe von Studienärzten und Sponsoren wie bei der Datenduplikation zugrunde liegen. Mehrere Untersuchungen haben darüber hinaus einen Zusammenhang zwischen Sponsoring durch die pharmazeutische Industrie und der höheren Wahrscheinlichkeit für ein publiziertes positives Studienergebnis herstellen können [37-39]. Neben einem für das eigene Produkt vorteilhaften Studiendesign (z.B. durch die Verwendung einer inadäquaten Dosierung des ausgewählten Vergleichspräparates [40-42]) oder einem Publikationsbias im eigentlichen Sinne (Nichtpublikation negativer Daten) könnte auch die selektive Darstellung von Studienergebnissen daran einen Anteil haben. Erste Analysen in der obenerwähnten Studie von Chan [32] scheinen dies tatsächlich zu bestätigen - aber sie zeigen auch, dass eine selektive Berichterstattung von Daten keineswegs nur ein durch die pharmazeutische Industrie verursachtes Problem ist. Wer auch immer zu einer Datenselektion beiträgt: er sorgt für eine eingeschränkte Verlässlichkeit von Nutzen/ Risiko- bzw. Kosten/Nutzen-Bewertungen. Über das Einfliessen solcher Darstellungen in Metaanalysen hinaus spielt auch ein sogenannter Referenzierungsbias (citation bias) eine Rolle. Ein weiteres Beispiel soll dies verdeutlichen: Die Helsinki-Heart-Studie wurde durchgeführt, um die mögliche Prävention einer koronaren Herzerkrankung durch den Lipidsenker Gemfibrozil zu untersuchen [43]. Die Ergebnisse in bezug auf die Primärprävention waren positiv, wurden 1987 isoliert publiziert [44] und in den drei Folgejahren 450mal zitiert. Eine Sekundärprävention konnte dagegen nicht aufgezeigt werden. Die Ergebnisse zu dieser Fragestellung erschienen erst sechs Jahre später [45] - in einem Journal mit geringer Verbreitung und zu einem Zeitpunkt, als sie von limitiertem Interesse waren, und wur- 
den in den drei Jahren danach 17mal zitiert [46]. Während den «positiven» Daten der Studie viel Aufmerksamkeit zugute kam, wurde von den «negativen» Daten nur wenig Notiz genommen. Es können berechtigte Zweifel an einer angemessenen und ausbalancierten Berücksichtigung dieser beiden Ergebnisse im medizinischen Alltag geäussert werden. Auch die obenzitierte Publikation der CLASS-Studie mit einem COX-2Inhibitor [33] - von welcher rund 30000 Sonderdrucke in Umlauf gebracht worden sind - wurde nachfolgend 169mal zitiert [34]. Ob die Öffentlichkeit von der anschliessenden Diskussion über die eingeschränkte Aussagefähigkeit der Studie genauso Anteil genommen hat wie an der primären Publikation, mag ebenfalls mit einem Fragezeichen versehen werden.

\section{Ausblick}

Was kann gegen diese Formen eines Publikationsbias unternommen werden? Im Sinne einer verstärkten Selbstkontrolle und Selbstregulierung sowohl der Ärzteschaft als auch der pharmazeutischen Industrie sollten diese Aspekte eines wissenschaftlichen Fehlverhaltens ebenfalls unmissverständlich in Kodizes bzw. interne Richtlinien aufgenommen werden. Ein Schritt in diese Richtung wurde durch Vertreter einiger pharmazeutischer Firmen mit der Erstellung der «Guidelines on Good Publication Practice for Pharmaceutical Companies» unternommen [47]. In diesen Richtlinien sind beispielsweise die Aspekte der Datenduplikation und akkuraten Wiedergabe von Studienergebnissen explizit angesprochen. Bisher hat sich jedoch nur eine beschränkte Anzahl pharmazeutischer Unternehmen zur Einhaltung dieser Richtlinien verpflichtet [48]. Die praktische Umsetzung steht und fällt damit, ob solche Richtlinien in konkrete firmeninterne Handlungsanweisungen (standard operating procedures) einfliessen oder nicht. So heisst es am Ende der «Guidelines on Good Publication Practice»: «Employees of ... [Namen der Firmen] contributed to the development of the Good Publication Practice document at various stages. However, the current guidelines may not necessarily represent the policies of these companies» [47]. Einzelne Unternehmen, wie z.B. Novartis, sind mit gutem Beispiel vorangegangen und haben bereits eigene klare Publikationsgrundsätze entwickelt [49].

Die Mehrheit der biomedizinischen Journals fordert heutzutage vom Autor - gemäss den «Uniform Requirements for Manuscripts Submitted to Biomedical Journals» [31] - eine Er- klärung, dass das eingereichte Manuskript bisher weder teilweise noch ganz an anderer Stelle veröffentlicht bzw. zur Veröffentlichung eingereicht wurde (Kongressbeiträge in der Regel ausgenommen). Das Problem der Redundanz in der wissenschaftlichen Literatur lässt sich damit jedoch nicht beseitigen - wie die Praxis zeigt. Strafmassnahmen, die von biomedizinischen Journals nach Bekanntwerden solcher Verstösse gegen die Publikationsrichtlinien verhängt werden, zum Beispiel die Ablehnung des duplizierten Artikels, die Veröffentlichung des Verstosses auf der Webseite des Journals oder die Nichtannahme weiterer Manuskripte eines Autors für eine definierte Zeitspanne, haben bisher nur eine mässige abschreckende Wirkung erzielt. Der Grund ist darin zu sehen, dass Verstösse in der Regel nur zufällig im Rahmen des Peer-reviewProzesses auffallen, zum Beispiel dann, wenn zwei Herausgeber den gleichen Experten als Begutachter einsetzen [24] oder wenn der Begutachter gezielt in Datenbanken nach bereits publizierten überlappenden Daten sucht.

Noch schwieriger ist der Umgang mit der selektiven Darstellung von Studienergebnissen bzw. der Änderung von Vorgaben im Studienprotokoll. Dieses Problem lässt sich seitens der Herausgeber der Zeitschriften nur dadurch lösen, wenn Studienprotokolle und auch alle Amendments zusammen mit dem Manuskript eingereicht, einem Peer review unterliegen und durch Veröffentlichung auf der Internetseite des Journals für jeden zugänglich werden. Solche Überlegungen findet man nicht nur wiederholt in der Literatur $[32,50,51]$, sondern werden von einigen Journals, wie z.B. dem Lancet [52], bereits heute praktiziert.

COPE nimmt, wie bereits zuvor erwähnt, Anzeigen von Publikationsverstössen entgegen [20]. Die Kompetenz zur Weiterverfolgung der Verstösse ist jedoch sehr eingeschränkt, so dass insbesondere in Grossbritannien immer wieder die Einrichtung eines nationalen Instituts mit legaler Kompetenz zur Verfolgung wissenschaftlichen Fehlverhaltens gefordert wurde [22, 53, 54]. In einzelnen Ländern, wie zum Beispiel in den USA, Australien oder Skandinavien, bestehen bereits seit längerem solche staatlichen Einrichtungen. In den USA wurde bereits vor 20 Jahren ein System installiert: Jede Forschungseinrichtung inner- und ausserhalb der USA, die öffentliche Gelder erhält, ist gesetzlich verpflichtet, wissenschaftliches Fehlverhalten zu verfolgen. Das seit 1989 bestehende Office of Research Integrity des US Department of Health \& Human Services überwacht heute die Verfahrensstandards der geförderten Einrichtungen sowie deren ein- 
geleitete Massnahmen [55]. Ein überführter Wissenschaftler kann bis zu 10 Jahre von Subventionen ausgeschlossen werden. Allerdings beschränken sich die verfolgten Fälle überwiegend auf schweres Fehlverhalten wie Datenfabrikation, Datenfälschung und Plagiat, was auch daran liegt, dass das Office of Research Integrity für die Verfolgung anderer Fälle über keine strafrechtliche Kompetenz verfügt [56]. Insgesamt 163 Anzeigen und 83 eröffnete Untersuchungen im Jahr 2002 [57] belegen die limitierte Bereitschaft, Fehlverhalten anzuzeigen, die eingeschränkte Kapazität bzw. legale Kompetenz des Offices sowie dessen mangelnde Effektivität in bezug auf «weniger schwere Verstösse» wie zum Beispiel Datenduplikation oder -selektion. Da die Situation in den skandinavischen Ländern vergleichbar ist, scheint ein Institut für wissenschaftliche Integrität in bezug auf eine Reduzierung des Publikationsbias daher kaum Bedeutung zu haben.

Als wirksamste Massnahme gegen den Publikationsbias ist eine vollständige Transparenz von Forschungsaktivitäten zu fordern. Vollständige Transparenz ist jedoch nur dadurch erzielbar, dass auf Studienprotokolle öffentlich und zentral an einer Stelle zugegriffen werden kann. Einmal mehr ist die Einrichtung eines Studienregisters zu fordern [58], wie es auch durch die anfangs erwähnten SAMW-Empfehlungen bereits 2002 im Hinblick auf die Schweiz getan wurde [9]: «Alle klinischen Versuche sind in einem zentralen Register zu erfassen. Die Erfassung bezweckt: die Duplikation von Versuchen zu vermeiden, nachträgliche, GCP-widrige Veränderungen am Versuchsprotokoll auszuschliessen und die Veröffentlichung der Ergebnisse zu gewährleisten. Das Register soll von einer geeigneten öffentlichen Institution geführt werden und öffentlich zugänglich sein [...].» Letztlich aber ist die Einrichtung eines internationalen Studienregisters erforderlich [58], durch welches weltweit klinische Studien obligat zu erfassen sind. Die Vielzahl bestehender nationaler Studienregister, wie zum Beispiel in den USA oder einzelnen europäischen Staaten, kann das Problem allein nicht lösen. Die Einrichtung zumindest eines gesamteuropäischen Registers wird seit Jahren gefordert [7, 59]. Zwar gibt es heute bereits Register, die alle Studien zu erfassen versuchen [60] - deren Limitationen liegen jedoch in der Freiwilligkeit der Registrierung durch die Studienverantwortlichen.

Was müsste unternommen werden und was würde sich konkret durch ein zentrales Studienregister ändern? Die obligate Eingabe der wichtigsten Kenngrössen der Studie und die damit verbundene Vergabe einer individuellen Stu- diennummer, wie es bereits heute bei der Registrierung im Current Controlled Trials metaRegister der Fall ist [60], würde weltweit die eindeutige Identifizierung einer Studie ermöglichen. Damit gingen die Studienverantwortlichen praktisch die Verpflichtung zur einmaligen, vollständigen und korrekten Bekanntmachung der Studienergebnisse ein. Die Pflicht zur Studienregistrierung wäre in behördlichen Richtlinien zu verankern. Ethikkommissionen könnten die Registrierung einer Studie als Bedingung für die Ausstellung eines positiven Votums voraussetzen. Und biomedizinische Zeitschriften sollten darüber hinaus nur Manuskripte von registrierten Studien zur Veröffentlichung annehmen.

\section{Literatur}

1 Krzyzanowska M, Pintilie M, Tannock IF. Factors associated with failure to publish large randomized trials presented at an oncology meeting. JAMA 2003;290:495-501.

2 Stern JM, Simes RJ. Publication bias: evidence of delayed publication in a cohort study of clinical research projects. Br Med J 1997;315:640-5.

3 Weber EJ, Callaham ML, Wears RL, Barton C, Young G. Unpublished research from a medical speciality meeting: why investigators fail to publish. JAMA 1998;280:257-9.

4 Rennie D. Thyroid storm. JAMA 1997;277:1238-43.

5 Dickersin K, Min YI, Meinert CL. Factors influencing publication of trial results. Follow-up of applications submitted to two institutional review boards. JAMA 1992;267:374-8.

6 Chalmers I. Underreporting research is scientific misconduct. JAMA 1990;263:1405-8.

7 Tonks A. A clinical trials register for Europe. Br Med J 2002;325:1314-5.

8 World Medical Association. Declaration of Helsinki (amended October 2000). www.wma.net/ e/policy/b3.htm.

9 Stauffacher W. Zusammenarbeit Ärzteschaft Industrie. Empfehlungen der Schweizerischen Akademie der Medizinischen Wissenschaften. Schweiz Ärztezeitung 2002;83:2165-71.

10 Schweizerische Gesellschaft für Chemische Industrie: Verhaltenskodex der pharmazeutischen Industrie in der Schweiz. www.sgci.ch/plugin/ template/sgci/*/11723.

11 Committee on Publication Ethics (COPE). Guidelines on good publication practice. JPGM 2000; 46:217-21.

12 Melander H, Ahlqvist-Rastad J, Meijer G, Beermann B. Evidence b(i)ased medicine - selective reporting from studies sponsored by pharmaceutical industry: review of studies in new drug applications. Br Med J 2003;326:1171-3.

13 Huston P, Moher D. Redundancy, disaggregation, and the integrity of medical research. Lancet 1996;347:1024-6. 
14 Huth EJ. Irresponsible authorship and wasteful publication. Ann Intern Med 1986;104:257-9.

15 Chen SA, Chiang C-E, Yang CJ, Cheng CC, Wu TJ, Wang SP, et al. Accessory pathway and atrioventricular node reentrant tachycardia in elderly patients: clinical features, electrophysiologic characteristics and results of radiofrequency ablation. J Am Coll Cardiol 1994;23:702-8.

16 Wu TJ, Chen SA, Chiang CE, Yang CJ, Cheng CC, Wang SP, et. al. Clinical features and electrophysiologic characteristics of accessory atrioventricular pathways and atrioventricular nodal reentrant tachycardia: comparative study between young and elderly patients. Am Heart J 1993;126:1341-8.

17 Mc Millen JI. Tolmetin sodium vs ibuprofen in rheumatoid arthritis patients previously untreated with either drug: a double-blind cross-over study. Curr Ther Res 1977;22:266-75.

18 McMillen JI. Rheumatoid arthritis: a double-blind study comparing tolmetin sodium with ibuprofen in patients with either drug previously. Curr Ther Res 1982;31:813-20.

19 Association of Medical Research Charities. Scientific integrity in medical research. www. amrc.org.uk/index.asp?id=937.

20 Committee on Publication Ethics. COPE Reports 2002 and 2003. www.publicationethics.org.uk/ cope2001/pages2001/reports.phtml.

21 National Research Foundation. The good, the bad and the ugly. S Afr J Sci 2003;99:402-3.

22 White C. Call for research misconduct agency. Br Med J 1998;316:1695.

23 Parmley WW. Redundant publication. J Am Coll Cardiol 1994;23:542-3.

24 Tobin MJ. AJRCCM's policy on duplicate publication. Infrequently asked questions (editorial). Am J Respir Crit Care Med 2002;166:433-4.

25 Oehm V, Lindner UK. Umstritten, aber etabliert der Impact Factor. Oder: Wie vermessen ist die Vermessung der Wissenschaft? Dtsch Ärzteblatt 2002;99:A1489-90.

26 Lenzen S. Nützlichkeit und Limitationen des sogenannten «Journal Impact Factor» bei der Bewertung von wissenschaftlichen Leistungen und Zeitschriften. Diabetes und Stoffwechsel $1997 ; 6: 273-5$

27 Seglen PO. Why the impact factor of journals should not be used for evaluating research. Br Med J 1997;314:498-502.

28 Tramér MR, Reynolds DJM, Moore RA, McQuay HJ. Impact of covert duplicate publication on meta-analysis: a case study. Br Med J 1997; 315:635-40.

29 Leizorovicz A, Haugh MC, Boissel JP. Metaanalysis and multiple publication of clinical trial reports. Lancet 1992;340:1102-3.

30 Von Elm E, Poglia G, Walder B, Tramér MR. Different patterns of duplicate publication. An analysis of articles used in systematic reviews. JAMA 2004;291:974-80.

31 International Committee of Medical Journal Editors (ICMJE). Uniform requirements for manuscripts submitted to biomedical journals. JAMA 1997;277:927-34 und www.icmje.org.
32 Chan A-W, Hrobjartsson A, Haahr MT, Gotzsche PC, Altman DG. Empirical evidence for selective reporting of outcomes in randomized trials. Comparison of protocols to published articles. JAMA 2004;291:2457-65.

33 Silverstein FF, Faich G, Goldstein JL, Simon LS, Pincus T, Whelton A, et al. Gastrointestinal toxicity with celecoxib vs nonsteroidal antiinflammatory drugs for osteoarthritis and rheumatoid arthritis: the CLASS study: a randomized controlled trial. JAMA 2000;284:1247-55.

34 Jüni P, Rutjes AWS, Dieppe PA. Are selective COX 2 inhibitors superior to traditional non steroidal anti-inflammatory drugs? Adequate analysis of the CLASS trial indicates that this may not be the case. Br Med J 2002;324:1287-8.

35 Berg Hrachovec J, Mora M. Reporting of 6-month vs 12 -month data in a clinical trial of celecoxib (letter to the editor). JAMA 2001;286:2398.

36 Wright JM, Perry TL, Bassett KL, Chambers GK. Reporting of 6-month vs 12-month data in a clinical trial of celecoxib (letter to the editor). JAMA 2001;286:2398-9.

37 Als-Nielsen B, Chen W, Gluud C, Kjargard LL. Association of funding and conclusions in randomized drug trials. A reflection of treatment effect or adverse events? JAMA 2003;290:921-8.

38 Kjaergard LL, Als-Nielsen B. Association between competing interests and authors' conclusions: epidemiological study of randomised clinical trials published in the BMJ. Br Med J 2002; 325:249-52.

39 Lexchin J, Bero LA, Djulbegovic B, Clark O. Pharmaceutical industry sponsorship and research outcome and quality: systematic review. Br Med J 2003;326:1167-70.

40 Bero LA, Rennie D. Influences on the quality of published drug studies. Int J Technol Assess Health Care 1996;12:209-37.

41 Rochon PA, Gurwitz JH, Simms RW, Fortin PR, Felson DT, Minaker KL, Chalmers TC. A study of manufacturer-supported trials of nonsteroidal anti-inflammatory drugs in the treatment of arthritis. Arch Intern Med 1994;154:157-63.

42 Schieppati A, Perico N, Remuzzi N. Conflict of interest as seen from a researcher's perspective. Science and Engineering Ethics 2002;8:337-42.

43 Manninen V. Clinical results with gemfibrozil and background to the Helsinki Heart Study. Am J Cardiol 1983;52:35B-38B.

44 Frick MH, Elo O, Haapa K, Heinonen OP, Heinsalmi P, Helo P, et al. Helsinki Heart Study: primary-prevention trial with gemfibrozil in middle-aged men with dyslipidemia. Safety of treatment, changes in risk factors, and incidence of coronary heart disease. N Engl J Med 1987 317:1237-45.

45 Frick MH, Heinonen OP, Huttunen JK, Koskinen P, Manttari M, Manninen V. Efficacy of gemfibrozil in dyslipidemic subjects with suspected heart disease. An ancillary study in the Helsinki Heart Study frame population. Ann Med 1993; $25: 41-5$ 
46 Egger M, Smith GD. Meta-analysis bias in location and selection of studies. Br Med J 1998;316:61-6.

47 Wager E, Field EA, Grossman L. Good publication practice for pharmaceutical companies. Curr Med Res Opin 2003;19:149-54.

48 Current endorsing and supporting companies of the Good Publication Practices Guidelines. www.gpp-guidelines.org.

49 Della Cioppa G, Garaud J-J. Registering clinical trials (letter to editor). JAMA 2003;290:2545-6.

50 Goldbeck-Wood S. Changes between protocol and manuscript should be declared at submission (commentary). Br Med J 2001;322:1460-1.

51 Chalmers I, Altman DG. How can medical journals help prevent poor medical research? Some opportunities presented by electronic publishing. Lancet 1999;353:490-3.

52 The Lancet. Information for authors. www. thelancet.com.

53 Smith R. The need for a national body for research misconduct. Br Med J 1998;316:1686-7.
54 Smith R. Meeting calls for a national body to respond to research misconduct. Br Med J 2001;323:889.

55 Office of Research Integrity. About ORI. www. ori.dhhs.gov.

56 Price A. Dealing with suspected misconduct. In: Proceedings of the retreat on the journal's role in scientific misconduct. www.councilscienceeditors. org/events/ori_retreat.pdf.

57 Office of Research Integrity. Annual Report 2002. www.ori.dhhs.gov/multimedia/acrobat/ORI Annual_Report_2002.pdf.

58 Dickersin K, Rennie D. Registering clinical trials. JAMA 2003;290:516-23.

59 Horton R, Smith R. Time to register randomized trials. Br Med J 1999;319:865-6.

60 Current Controlled Trials. MetaRegister of controlled trials. www.controlled-trials.com. 